\title{
Island constraints are not the result of sentence processing
}

\author{
Helen Goodluck, Frank Tsiwah \& Kofi Saah*
}

\begin{abstract}
On the basis of a comparison between sentence judgements in English vs. Akan, we argue that island constraints (positions to which a question word can be linked) cannot be reduced to the effects of the sentence processing mechanism. Questions that violate constraints are judged better in Akan than in English, although the challenge to the sentence processor is the same for the two languages. We argue specifically that the constraints cannot be reduced to the effects of specificity of the question phrase, and must be attributed to two different grammatical mechanisms for the languages.
\end{abstract}

Keywords. Island constraints; processing; English; Akan

1. Introduction. English and Akan differ in the range of questions that they allow. English is subject to 'island constraints', which block questions from referring (inter alia) to a position inside a complex NP (1a), an adjunct clause (2a) or an embedded question (3a). All the equivalent questions in Akan (1b, $2 b$ and $3 b)$ are grammatical.

(1) a. *What did you see a person that cut?

b. Den na wo huu onipa ko a otawaae?

What FOC you see person SP REL 3SG:cut [e]

$(\mathrm{FOC}=$ focus marker; $\mathrm{SP}=$ specificity marker; $\mathrm{REL}=$ relativizer $)$

(2) a. *What did Ama read the Graphic before she wrote?

b. Den na Ama kanee Graphic ansa na orekyercw?

What FOC Ama read the Graphic before 3SG:wrote [e]

(3) a. *Who did the old lady remember what she should buy for?

b. Hwan na aberewa no kae se den na $\varepsilon w \supset$ se

Who FOC old lady the remember that what FOC should that 3SG:buy give him/her

Saah and Goodluck (1995) analyse the difference between English and Akan as a difference between movement in English and pronominal binding in Akan. The pronoun is phonetically null in (1b) and (2b) and overt in (3b). In Chomskyan generative grammar, only movement operations result in island effects.

There has been a long-standing debate about whether restrictions on question formation and other sentence types in English and similar languages must be attributed to the particular grammatical mechanism at hand, or can be reduced to the pressures of sentence processing. Among the more recent articles contributing to the debate is that of Hofmeister and Sag (2010). Hofmeister and Sag found that a d(iscourse)-linked wh-phrase, such as which convict led to faster reaction times and higher judgement ratings for sentences that violated the complex NP constraint (CNPC) and the Wh-island constraint than a simple question phrase (who) did, a fact that they attribute to a stronger memory trace for discourse-linked (+DL) question phrase. They

\footnotetext{
* Authors: Helen Goodluck, University of York, United Kingdom (helen.goodluck@york.ac.uk), Frank Tsiwah, University of Groningen, The Netherlands (f.tsiwah@rug.nl) and Kofi Saah, University of Ghana, Ghana (kofisaah@hotmail.com). We would like to thank Mary Edward for collecting the data in Ghana and Peter Sells and attendees at the LSA meeting for helpful discussions of the content of our poster.
} 
imply that island constraints are the result of the human sentence processing mechanism, and do not arise from particular grammatical mechanisms. Our study investigates this claim with a cross-linguistic comparison between English and Akan. These languages offer a particularly fruitful potential hunting ground, since the word order is the same with minor exceptions in both languages, and yet the judgements have been found to be strikingly different for violations of island constraints (Saah and Goodluck 1995).

2. Method. We asked participants in two experiments to judge sentences on a scale of 0 (bad) 5 (good). The materials were presented in written form, and participants were given as long as they wished to complete the questionnaire. There were 18 participants in each language (English and Akan) in each experiment; all participants were undergraduates at the University of York (English speakers) and the University of Ghana (Akan speakers), blind to the purpose of the study. In both experiments, a +DL question phrase was compared to a -DL question phrase. Four tokens of each sentence type were included in each questionnaire. Experiment 1 tested the CNPC and the adjunct constraint. Experiment 2 tested the Wh-island constraint. Both experiments included grammatical and ungrammatical sentences as fillers and, in experiment 1, an independent test of use of the grammar of the language (see 10 below). ${ }^{\dagger}$ All materials were Latin squared, such that no participant received the same basic sentence frame in more than one condition. The sentence types tested are summarised as follows:

\section{Experiment 1}

Base condition (declarative) CNPC

(4) a. Martin called the woman that sells flowers

b. Kofi freq maame no a ston flawes no

Kofi call:PST woman the REL 3SG:sell flowers CD

$(\mathrm{CD}=$ Clause determiner $)$

CNPC violation with definite head NP

a. What/Which flowers did Martin call the woman that sells?

b. Den/Den flawes na Kofi frec maame no a ston no?

What/Which flowers FOC Kofi call:PST woman the REL 3SG:sell CD

(6) a. Ken got a license before he drove the car

b. Ken nyaa license ansa na ootwi car no

Ken get:PST licence before 3SG:drive car the

Adjunct island violation

(7) a. What/which car did Ken get a license before he drove?

b. Den/Den car na Ken nyaa license ansa na ootwi no?

What/Which car FOC Ken get:PST license before 3SG:drive CD

\footnotetext{
${ }^{\dagger}$ In experiment 1 , in separate conditions to the main CNPC test (5a,b below), an indefinite head was compared to a definite head. No effect of definiteness of the head was found, consistent with the results of Tollan and Heller (2015), and contrary to the weak effect of indefinite heads being easier found in Hofmeister and Sag (2010).
} 


\section{Experiment 2}

Base condition (questioning into a non-interrogative clause)

(8) a. Who did the clever doctor remember that he could send the results to?

b. Hwan na dokota benefo no kae se obetumi de ensunsuansos no Who FOC doctor clever the remember that 3SG:can take result the amane no?

send $\mathrm{CD}$

Wh-island violation

(9) a. Who/Which expert did the clever doctor remember what he could send to?

b. Hwan/Den okunini na dokota benefo no kae se den na obetumi de Who/Which expert FOC doctor clever the remember:PST that what FOC 3SG:can amane no?

send $\mathrm{CD}$

3. Results. The mean score (/5) for each experiment is given in Tables 1 and 2. An ordered logistic regression was used for the analysis using the polr function of the MASS library of R (version 3.2.5). Table 1 shows that there is a large difference between the two languages in the acceptability of questions that penetrate a complex NP, and for questions that penetrate an adjunct clause (CNPC: $\beta=-2.91, \mathrm{SE}=0.33, \mathrm{t}=-2.79, \mathrm{p}=.001$; Adjunct: $\beta=1.65, \mathrm{SE}=0.21, \mathrm{t}=$ $6.02, \mathrm{p}=.001)$. There is an effect of $+/$-DL for CNPC $(\beta=0.86, \mathrm{SE}=0.31, \mathrm{t}=-2.79, \mathrm{p}=.005)$, with $+\mathrm{DL}$ items scoring higher than -DL. There is no interaction between $+/$-DL and language (CNPC, $\mathrm{p}=.11$; Adjunct, $\mathrm{p}=.83$ ). We combined the +DL and -DL conditions for each language and compared them using a Mann-Whitney U test. The result was significant ( $\mathrm{p}<$ .00001) by both participant and by item.

\begin{tabular}{lcccccc}
\hline & \multicolumn{3}{c}{ English } & \multicolumn{3}{c}{ Akan } \\
\hline & Base & $-\mathrm{DL}$ & $+\mathrm{DL}$ & Base & $-\mathrm{DL}$ & $+\mathrm{DL}$ \\
CNP Constraint & 4.49 & 0.75 & 0.90 & 3.97 & 2.76 & 3.54 \\
$\begin{array}{l}\text { Adjunct } \\
\text { Constraint }\end{array}$ & 4.91 & 0.82 & 1.17 & 4.33 & 3.30 & 3.70 \\
\hline
\end{tabular}

Table 1. Mean scores Experiment 1

It is tempting to suppose that for whatever reasons Akan speakers are more willing to judge sentences as better than their English counterparts. However, this is not a generalization that holds over the entire study. In Akan, an overt pronoun is ungrammatical in sentence final position,

Ber a tractor no yec dada no okuani no tonn $[\mathrm{e}] / *$ no

When tractor the become:PST old CD farmer the sell:PST

'When the tractor was too old, the farmer sold it'

In English, the opposite is true - the translation of (10) is ungrammatical without the final pronoun. The mean scores for sentences with an overt pronoun at the end were lower for Akan than for English and significantly different $(\mathrm{Akan}=3.19$, English $=4.68 ; \beta=1.92, \mathrm{SE}=0.44, \mathrm{t}=$ $6.70, \mathrm{p}=.001$ ). Thus Akan speakers were not in general predisposed to judge sentences more liberally than English speakers. 
In experiment 2, again there is a large difference between languages, with Akan speakers having higher scores for sentences that violated the $\mathrm{Wh}$-island constraint $(\beta=-1.44, \mathrm{SE}=0.33, \mathrm{t}$ $=-4.44, \mathrm{p}=.001)$. We combined the $+\mathrm{DL}$ and $-\mathrm{DL}$ conditions for each language and compared them using a Mann-Whitney $U$ test. The result was significant by item ( $p<.0001)$, but fell short of significance by participant $(U=99.5$; critical value for $p<.05=99)$. Five Akan-speaking participants had a score of 5 or less out, of a range of $0-33$ ).

\begin{tabular}{lcccccc}
\hline & \multicolumn{3}{c}{ English } & \multicolumn{3}{c}{ Akan } \\
\hline & Base & $-\mathrm{DL}$ & $+\mathrm{DL}$ & Base & -DL & $+\mathrm{DL}$ \\
Wh-island & 3.40 & 0.96 & 0.91 & 3.51 & 2.45 & 1.60 \\
\hline
\end{tabular}

Table 2. Mean scores Experiment 2

In this experiment we obtained a result that we did not predict, i.e. questions with -DL phrases show an advantage over those with $+\mathrm{DL}$ phrases $(\beta=0.95, \mathrm{SE}=0.33, \mathrm{t}=2.88, \mathrm{p}=.004)$. This is potentially explicable in terms of the target positions for the wh-phrase. In both experiment 1 and experiment 2, the target (correct) position was an object position. However, the question phrase was inanimate in experiment 1 (What, Which flowers/Den, Den flawes) and animate in experiment 2 (Who, Which expert/Hwan, Den okunini). The animate question phrase may have set up an expectation of a subject gap, which may have been greater with a lexically specified wh-phrase. When the subject position in the embedded sentence is occupied and the expectation of a subject gap is not fulfilled, the disruption caused will be greater for a + DL phrase, and hence the scores will be lower. This result runs contrary to that of Hofmeister and Sag (2010), who used animate wh-phrases and did find +DL phrases were processed more easily than -DL phrases when combined with an object gap.

4. Discussion. Our results add to the literature arguing that, as opposed to the position of Hofmeister and Sag and others, constraints on question formation cannot be reduced to the sentence processing mechanism (see Sprouse et al. 2012a,b, Toller and Heller 2015, Yoshida et al. 2014). The challenge posed by English and Akan in processing a question is basically the same: to find a position for a sentence initial question phrase. The effects of D-linking are similar in the two languages, and do not interact with the effect of islands. In processing oral input, Akan like English seeks to find the earliest possible location in the incoming string for the question phrase, in accord with the Active Filler Strategy (Frazier \& Flores d'Arcais 1989; see data in Saah and Goodluck 1995). However, in more reflective judgement tasks, the desire to find a gap in which to location the question word may be lessened and the grammatical mechanism of the language may override the need to fill a gap as soon as possible. We attribute the overall higher scores in Akan to the use of a different grammatical mechanism (pronominal binding, rather than movement) when an island-creating boundary is crossed. We did find in Experiment 2 that a minority of Akan-speaking participants had low scores for the island-violating questions, which we attribute to the cognitive complexity of embedding a question within a question, rather than to a difference in the syntactic mechanism used. Thus we adopt a position similar to Truswell (2011): there is a basic effect of syntactic mechanism used, which may be overlaid by other factors such as D-linking, and cognitive complexity in processing, but which cannot be replaced by such factors. 


\section{References}

Frazier, Lyn and Giovanni Flores d'Arcais 1989. Filler-driven parsing: A study of gap filling in Dutch. Journal of Memory and Language 28, 311-344. https://doi.org/10.1016/0749- 596X(89)90037-5.

Hofmeister, Philip; Laura S. Casasanto; and Ivan Sag. 2012. How do individual differences relate to the acceptability judgements? A reply to Sprouse, Wagers and Philips. Language 88, 390-400. https://doi.org/10.1353/lan.2012.0004.

Saah, Kofi. 1992. Null object construction in Akan. MITWPL 17.

Saah, Kofi and Helen Goodluck. 1995. Island effects in parsing and grammar: Evidence from Akan. The Linguistic Review 12, 381-409. https://doi.org10.1515/tlir.1995.12.4.381.

Sprouse, Jon; Matt Wagers; and Colin Philips. 2012a. A test of the relation between working memory capacity and syntactic island effects. Language 88, 82-123. DOI: https://doi.org/10.1353/lan.2012.0004.

Sprouse, Jon; Matt Wagers and Colin Philips. 2012b. Working memory capacity and island effects: A reminder of the issues and the facts. Language 88, 401-407. https://doi.org/10.1353/lan.2012.0029.

Tollan, Rebecca and Daphne Heller 2015. Elvis Presley on an island: wh dependency formation inside complex NP objects. Proceedings of NELS 46.

Truswell, Robert. 2011. Events, Phrases and Questions. Oxford: Oxford University Press.

Yoshida, Masaya; Nina Kazanina; Leticia Pablos; and Patrick Sturt. 2014. On the origin of islands. Language, Cognition and Neuroscience, 29, 761-770. https://doi.org/10.1083/01690965.2013.788196. 